\title{
Hadronic uncertainties and isospin violation in supersymmetric dark matter models
}

\author{
Lewis C. Tunstall* \\ Albert Einstein Center for Fundamental Physics, \\ Institute for Theoretical Physics, University of Bern, \\ Sidlerstrasse 5, CH-3012 Bern, Switzerland \\ E-mail: tunstalleitp.unibe.ch \\ Andreas Crivellin \\ CERN Theory Division, \\ CH-1211 Geneva 23, Switzerland \\ Martin Hoferichter \\ Institute for Nuclear Theory, University of Washington, \\ Seattle, WA 98195-1550, USA \\ Massimiliano Procura \\ Fakultät für Physik, Universität Wien, \\ Boltzmanngasse 5, A-1090 Vienna, Austria
}

\begin{abstract}
Current limits from dark matter direct-detection experiments place a powerful constraint on the parameter space of the Minimal Supersymmetric Standard Model (MSSM). The interpretation of these limits, however, depends sensitively on the hadronic uncertainties associated with the scattering of supersymmetric dark matter particles off nucleons. For spin-independent scattering, we review the role of 2- and 3-flavour chiral perturbation theory in the determination of these hadronic uncertainties, and quantify the amount of isospin violation within several simplified versions of the MSSM. In each case, we identify parameter-space configurations that produce (almost) vanishing cross sections and examine the complementarity of constraints due to directdetection, flavour, and collider experiments. In the vicinity of these so-called blind spots, we find that isospin violation may be much larger than typically expected in the MSSM.
\end{abstract}

The 8th International Workshop on Chiral Dynamics, CD2015

29 June 2015 - 03 July 2015

Pisa, Italy

\footnotetext{
* Speaker.
} 


\section{The Dark Matter Puzzle}

There is nowadays a consensus of evidence from astrophysical observations [1,2] that the total matter density in the Universe $\Omega_{\mathrm{M}} \approx 0.3$ is dominated by a component of non-baryonic origin. The data imply that this exotic form of matter is cold, non-luminous (i.e. "dark"), and has a present-day density of $\Omega_{\mathrm{DM}} \approx 0.25$. Among several possible candidates, weakly interacting massive particles (WIMPs) have received considerable attention from both theorists and experimentalists.

The appeal of WIMP dark matter stems from the observation that the contribution to $\Omega_{\mathrm{DM}}$ from a massive particle $\chi$ is determined (up to logarithmic corrections) by the $\chi \chi$ annihilation cross-section $\sigma_{\text {ann }}$ into lighter particles,

$$
\Omega_{\chi} \approx \frac{10^{-13} \text { barn }}{\left\langle\sigma_{\mathrm{ann}} v\right\rangle}
$$

where $v$ is the relative velocity of the incoming $\chi$ s and $\langle\ldots\rangle$ denotes a thermal average. If $\chi$ has weak-strength interactions, then $\left\langle\sigma_{\mathrm{ann}} v\right\rangle$ is of the order of a picobarn and $\Omega_{\chi}$ can reproduce part or all of the observed relic density $\Omega_{\mathrm{DM}}$. Taken at face value, ${ }^{1}$ this so-called WIMP miracle provides a surprising connection between dark matter and the electroweak scale, where solutions to the hierarchy problem of the Standard Model (SM) are expected to give rise to new physics.

An explicit realisation of this picture occurs in the Minimal Supersymmetric SM (MSSM), where softly-broken supersymmetry stabilises the electroweak scale, while an exact $R$-parity ensures that the lightest superpartner (LSP) is a stable neutralino (also denoted by $\chi$ ) with electroweak couplings and a mass in the range from tens to hundreds of $\mathrm{GeV}$. If dark matter is composed of neutralinos, then galactic rotation curves imply there should be a considerable flux of these particles in the Milky Way. This opens up the possibility of detecting neutralinos directly using terrestial experiments, where e.g. the elastic scattering of neutralinos off nuclei may leave measurable imprints in the recoil energy of the target. Although no conclusive signal has yet been found in such direct detection experiments, the current exclusion limits from LUX [4], as well as upcoming proposals like XENON1T [5] and LUX-ZEPLIN (LZ) [6], can lead to strong constraints on the parameter space of the MSSM. The interpretation of these limits, however, depends sensitively on the hadronic uncertainties associated with the $\chi$-nucleon scattering cross-section.

Here we review the role of 2- and 3-flavour chiral perturbation theory in the determination of these hadronic uncertainties (Sec. 2), and quantify the amount of isospin violation within several simplified models of the MSSM (Sec. 3). This is a summary of a general analysis [7] concerning parameter space regions where the $\chi$-nucleon scattering cross-section is strongly suppressed. A key feature of our work is that in the vicinity of these so-called blind spots $[8,9]$, isospin-violation may be much larger than typically expected in the MSSM. Concluding remarks are given in Sec. 4.

\section{Hadronic Matrix Elements and Neutralino-Nucleus Scattering}

In general, the elastic scattering of the LSP off a nucleus $\mathscr{N}={ }_{Z}^{A} X$ can involve spin-dependent and spin-independent (SI) interactions. Focusing on the latter, the key observable of interest is the

\footnotetext{
${ }^{1}$ There are well known cases [3] where the estimate in (1.1) no longer applies.
} 
$\chi-\mathscr{N}$ scattering cross-section at zero momentum transfer

$$
\sigma_{\mathrm{SI}}=\frac{4 \mu_{\chi}^{2}}{\pi}\left[Z f_{p}+(A-Z) f_{n}\right]^{2},
$$

where $\mu_{\chi}=m_{\chi} m_{\mathscr{N}} /\left(m_{\chi}+m_{\mathscr{N}}\right)$ is the reduced mass of the $\chi-\mathscr{N}$ system, and $f_{p}$ and $f_{n}$ are effective SI couplings of the LSP to the proton and neutron respectively. For nucleons $N$, the $\chi-N$ couplings $f_{N}$ are defined by

$$
\frac{f_{N}}{m_{N}}=\sum_{q=u, d, s} f_{q}^{N} C_{q}+f_{Q}^{N} \sum_{q=c, b, t} C_{q},
$$

where $C_{q}$ is the Wilson coefficient of the scalar operator $m_{q} \bar{\chi} \chi \bar{q} q$ while

$$
m_{N} f_{q}^{N}=\left\langle N\left|m_{q} \bar{q} q\right| N\right\rangle \quad \text { and } \quad f_{Q}^{N}=\frac{2}{27}\left(1-f_{u}^{N}-f_{d}^{N}-f_{s}^{N}\right) .
$$

Traditionally [10-12,30], the scalar matrix elements of the light quarks (2.3) have been determined by applying the Feynman-Hellmann theorem

$$
\left\langle N\left|m_{q} \bar{q} q\right| N\right\rangle=m_{q} \frac{\partial m_{N}}{\partial m_{q}}
$$

to the chiral $S U(3)_{L} \times S U(3)_{R}$ expansion of the nucleon mass

$$
m_{N}=m_{0}+\sum_{\phi=\pi, K, \eta} c_{\phi} m_{\phi}^{2}+O\left(m_{\pi, K, \eta}^{3}\right),
$$

where $m_{0}$ is the chiral-limiting value of $m_{N}$ and $c_{\phi}$ is a linear combination of low-energy constants. Then the couplings $f_{q}^{N}$ can be expressed in terms of the pion-nucleon sigma-term

$$
\sigma_{\pi N}=\langle N|\bar{m}(\bar{u} u+\bar{d} d)| N\rangle, \quad \bar{m}=\frac{1}{2}\left(m_{u}+m_{d}\right),
$$

the strangeness content of the nucleon

$$
y=\frac{2\langle N|\bar{s} s| N\rangle}{\langle N|\bar{u} u+\bar{d} d| N\rangle}
$$

and another parameter

$$
z=\frac{\langle N|\bar{u} u-\bar{s} s| N\rangle}{\langle N|\bar{d} d-\bar{s} s| N\rangle}
$$

that is related to isospin violation. For some time, it has been known that obtaining reliable uncertainty estimates in this framework is problematic because:

1. The up- and down-quark coefficients $f_{u, d}^{N}$ depend explicitly on the 3-flavour quantities $y$ and $z$. As a result, the order $30 \%$ uncertainties associated with chiral $S U(3)_{L} \times S U(3)_{R}$ perturbation theory $\chi \mathrm{PT}_{3}$ propagate into the 2 -flavour sector;

2. The strange-quark scalar matrix element is defined as

$$
f_{s}^{N}=\frac{\sigma_{\pi N}}{2 m_{N}} \frac{m_{s}}{\bar{m}} y,
$$


where $m_{s} / \bar{m}=(27.4 \pm 0.4)$ [14], the strangeness content is taken from the relation $y=$ $1-\sigma_{0} / \sigma_{\pi N}$, with $\sigma_{0}=(36 \pm 7) \mathrm{MeV}$ [15], and $z \simeq 1.49$ is extracted from leading-order fits to the baryon mass spectrum [16]. Within the range $\sigma_{\pi N}=50 \pm 15 \mathrm{MeV}$ covered in [17-22], large values for the strangeness content of the nucleon ${ }^{2}$ and the corresponding coupling $f_{s}^{N}=$ $0.2 \pm 0.2$ have been inferred. Although still employed in the literature (see e.g. [24,25]), such large values are incompatible with modern lattice QCD calculations, which yield a much smaller and more reliable determination of $f_{s}^{N}$ [26]:

$$
\left.f_{s}^{N}\right|_{\text {lattice }}=0.043 \pm 0.011
$$

Evidently, the choice of input for $f_{s}^{N}$ can have a dramatic effect on the interpretation of experimental limits on $\sigma_{\mathrm{SI}}$. For example, it has been shown [27] that lattice input for $f_{s}^{N}$ can weaken bounds on the constrained MSSM parameter space by factors of 5-10 (!) relative to those obtained in the traditional $\chi \mathrm{PT}_{3}$ framework.

In Ref. [28], it was demonstrated that problem 1 can be circumvented by using the 2-flavour theory $\chi \mathrm{PT}_{2}$ directly, thus avoiding the 3 -flavour expansion in the first place. (Problem 2 is avoided by adopting lattice input (2.10) for $f_{s}^{N}$.) Starting from the $\chi \mathrm{PT}_{2}$ expansion of the nucleon mass at $O\left(m_{\pi}^{3}\right)$ and including the effects due to strong isospin violation, one finds [28]

$$
\begin{aligned}
f_{u}^{N} & =\frac{\sigma_{\pi N}(1-\xi)}{2 m_{N}}+\Delta f_{u}^{N}, & f_{d}^{N} & =\frac{\sigma_{\pi N}(1+\xi)}{2 m_{N}}+\Delta f_{d}^{N}, \\
\Delta f_{u}^{p} & =(1.0 \pm 0.2) \times 10^{-3}, & \Delta f_{u}^{n} & =(-1.0 \pm 0.2) \times 10^{-3} \\
\Delta f_{d}^{p} & =(-2.1 \pm 0.4) \times 10^{-3}, & \Delta f_{d}^{n} & =(2.0 \pm 0.4) \times 10^{-3},
\end{aligned}
$$

where

$$
\xi=\frac{m_{d}-m_{u}}{m_{d}+m_{u}}=0.36 \pm 0.04
$$

is taken from [14]. A key feature of this approach is that isospin violation can be systematically accounted for, so it becomes natural to ask whether there exist regions in the MSSM parameter space where such effects may be important. In this context, we observe that the cross section (2.1) may be rewritten as ${ }^{3}$

$$
\sigma_{\mathrm{SI}}=\frac{4 \mu_{\chi}^{2}}{\pi} f_{p}^{2}\left[A+(A-Z)\left(\frac{f_{n}}{f_{p}}-1\right)\right]^{2},
$$

so that the departure of $f_{n} / f_{p}$ from unity emerges as a convenient measure of isospin violation.

\section{Blind Spots and Isospin Violation}

Virtually nothing is known about how supersymmetry is broken at the weak-scale, so in general, the MSSM contains more than 100 parameters whose values are to be constrained by experiment. Given the huge parameter space, it is necessary to make additional, simplifying assumptions

\footnotetext{
${ }^{2}$ Although see [23] for important higher-order corrections to $\sigma_{0}$ which can reduce the value of $y$, albeit with large uncertainties.

${ }^{3}$ Corrections to the single-nucleon picture underlying (2.1) in the form of two-nucleon currents can be systematically taken into account using effective field theory [29-32].
} 

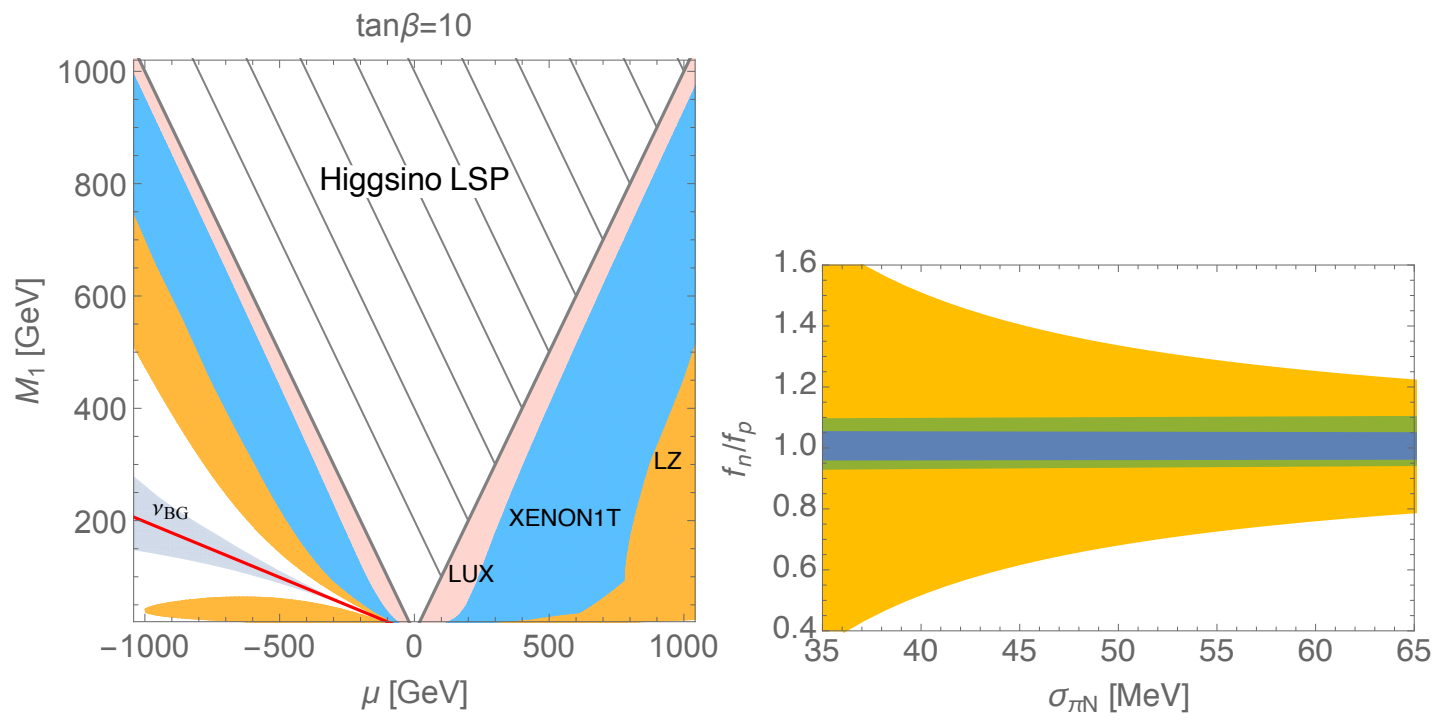

Figure 1: Left: Current and projected limits on SI $\chi$-xenon scattering due to $h$ exchange with $\tan \beta=10$. The pink band shows the existing constraints from LUX [4], while projected limits from XENON1T [5] and LZ [6] are given by the blue and orange regions respectively. The blind spot where the SI cross section vanishes is denoted by the red line and lies within the irreducible neutrino background $\left(v_{\mathrm{BG}}\right)$ shown in grey. Right: Amount of isospin violation in terms of $f_{n} / f_{p}$. The coloured bands correspond to the $1 \sigma$ uncertainties associated with the different determinations of the scalar matrix elements $f_{q}^{N}$ discussed in the text.

in order to undertake phenomenological analyses. In our work [7], we have examined several simplified models where all but a few superpartners are decoupled from the spectrum. Not only does this allow us to focus on dark matter signals of interest, it also allows us to develop an analytical framework with which to explore the underlying parameter space. Here we consider the effects of isospin violation in two simplified models $[8,9]$ where $\chi$-quark scattering is mediated by Higgs exchange in the $t$-channel: for an analysis of scenarios involving the exchange of light $3^{\text {rd }}$ generation squarks, we refer the reader to [7].

In these models, the lightest neutralino $\chi$ is an admixture of bino, wino, and Higgsino interaction eigenstates, obtained by diagonalising the mass matrix

$$
\left(\begin{array}{cccc}
M_{1} & 0 & -\frac{1}{2} g_{1} v_{d} & \frac{1}{2} g_{1} v_{u} \\
0 & M_{2} & \frac{1}{2} g_{2} v_{d} & -\frac{1}{2} g_{2} v_{u} \\
-\frac{1}{2} g_{1} v_{d} & \frac{1}{2} g_{2} v_{d} & 0 & -\mu \\
\frac{1}{2} g_{1} v_{u} & -\frac{1}{2} g_{2} v_{u} & -\mu & 0
\end{array}\right) .
$$

Here $M_{1}\left(M_{2}\right)$ are the soft supersymmetry-breaking masses of the bino (wino), $\mu$ is the Higgsino mass parameter, and $v_{u, d}$ are the two Higgs $H_{u, d}$ vacuum expectation values, whose ratio $v_{u} / v_{d}$ is denoted by $\tan \beta$. Let us now consider the two simplified models in turn.

Model 1 : The simplest possible dark matter model in the MSSM consists of a light Higgs $h$ with properties consistent with the SM, and a neutralino LSP which we assume to be predominantly bino-like, i.e. where $M_{1} \ll M_{2}, \mu$. In this case, the corresponding Wilson coefficients are

$$
C_{u_{i}}^{h}=C_{d_{i}}^{h}=\frac{g_{1}^{2}}{4 m_{h}^{2}} \frac{1}{M_{1}^{2}-\mu^{2}}\left(M_{1}+\mu \sin 2 \beta\right), \quad u_{i}=u, c, t, \quad d_{i}=d, s, b,
$$



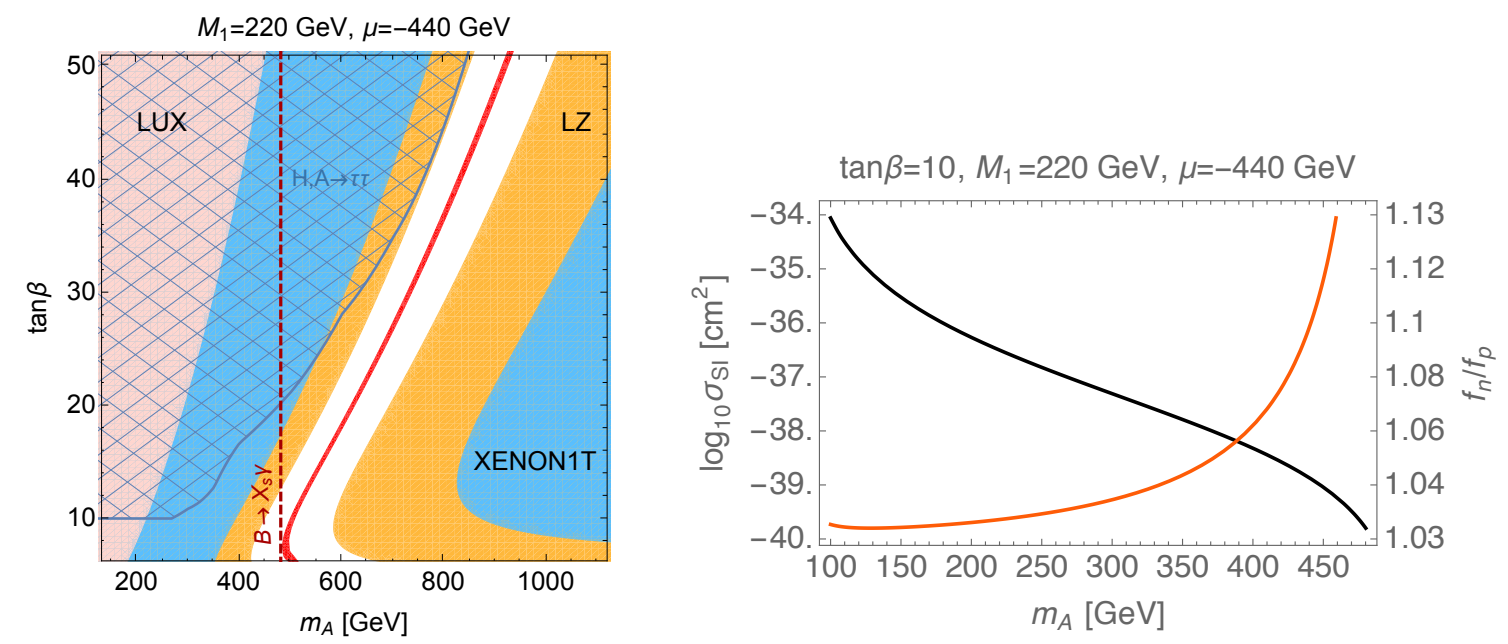

Figure 2: Left: Current and projected limits on SI $\chi$-xenon scattering due to $h, H$ exchange with different benchmark values for $M_{1}$ and $\mu$. Excluded regions and the blind spot are colour-coded as in Fig. 1, with the cross-hatched region in dark-blue corresponding to CMS limits [33] on $H, A \rightarrow \tau^{+} \tau^{-}$. The region to the left of the dark-red dashed line at $m_{A} \simeq m_{H^{+}} \simeq 480 \mathrm{GeV}$ is excluded by $B \rightarrow X_{s} \gamma$ [34]. Right: Pseudoscalar Higgs mass $m_{A}$ dependence of the SI $\chi$-xenon cross section (black) and the central value of $f_{n} / f_{p}$ (red) as determined by $\chi \mathrm{PT}_{2}$ method.

so we conclude that (a) for $\mu$ negative, the SI amplitude vanishes in the parameter space regions where $M_{1}+\mu \sin 2 \beta=0$ [8]; (b) the $C_{q_{i}}$ are independent of quark flavour, so isospin violation is determined entirely by hadronic quantities

$$
\frac{f_{n}}{f_{p}}=\left(\frac{m_{n}}{m_{p}}\right) \frac{2+7 \sum f_{q}^{n}}{2+7 \sum f_{q}^{p}}
$$

Note in particular that $f_{s}^{N}$ dominates the sums in (3.3) and can produce the largest source of uncertainty on $f_{n} / f_{p}$. In the left plot of Fig. 1 , we show the prospects for constraining the blind spot with current and - assuming no signal is seen - future direct detection experiments. In the right plot, we display the $1 \sigma$ uncertainties on $f_{n} / f_{p}$ as a function of $\sigma_{\pi N}$. Here we have compared three approaches to determine the scalar matrix elements:

(1) the $\chi \mathrm{PT}_{2}$ method (blue) proposed in [28], with $f_{s}^{N}$ taken from the lattice (2.10);

(2) the traditional $\chi \mathrm{PT}_{3}$ method $[10-12,30]$ (orange) where $f_{s}^{N}$ is typically large;

(3) use of the $\chi \mathrm{PT}_{3}$ formalism, but with lattice input for $f_{s}^{N}$ (green).

As seen in the Figure, the uncertainties associated with the traditional $\chi \mathrm{PT}_{3}$ method are large and display a strong sensitivity to $\sigma_{\pi N}$. In contrast, the $\chi \mathrm{PT}_{2}$ method is largely independent of $\sigma_{\pi N}$ and yields uncertainties which are smaller by factors of $\approx 6$ or more. In this case, the $1 \sigma$ uncertainties on $f_{n} / f_{p}$ are at the $5 \%$ level, so the naive expectation that isospin violation is a small effect in the MSSM is borne out explicitly.

Model 2 : A more interesting example arises when the spectrum is extended to include the heavier Higgs states $H, A, H^{ \pm}$of the MSSM. In this case, destructive interference between the $h$ 

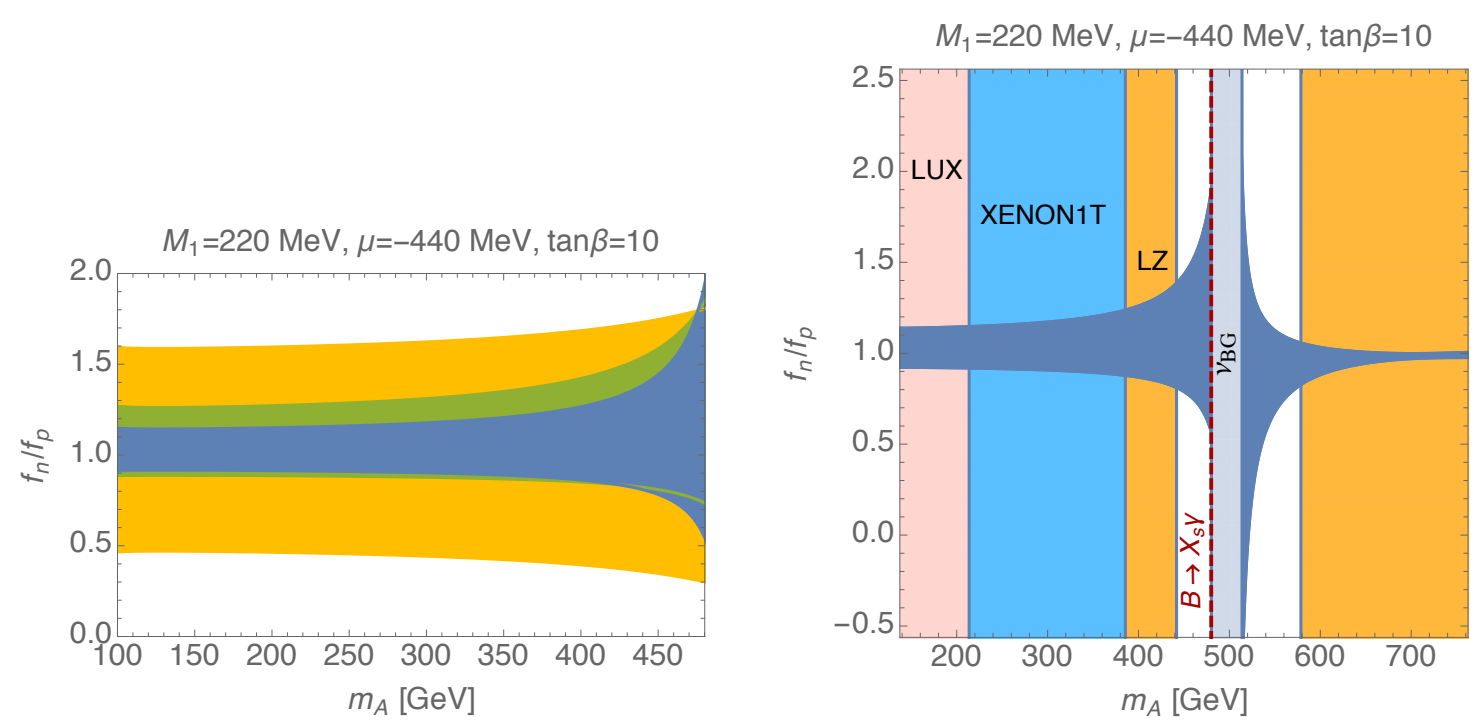

Figure 3: Left: Amount of isospin violation in terms of $f_{n} / f_{p}$ due to $h, H$ exchange in $\chi$-xenon scattering. The shaded regions show the uncertainty on $f_{n} / f_{p}$ due to each determination of the scalar matrix elements discussed in the text. Colour coding as in Fig. 1.

and $H$ amplitudes gives rise to a generalised blind spot [9]

$$
\frac{2}{m_{h}^{2}}\left(M_{1}+\mu s_{2 \beta}\right)+\mu \tan \beta \frac{1}{m_{H}^{2}} \simeq 0
$$

for $\tan \beta$ large and $m_{A}>m_{h}$. As a result of the extended Higgs sector, a complementarity of constraints from collider, flavour and direct detection arises (left plot of Fig. 2). In particular, increased sensitivity in future LHC searches for the decay $A, H \rightarrow \tau^{+} \tau^{-}$will be able to cover the blind spot region (3.4), where direct detection limits are necessarily weak.

Another interesting feature of (3.4) is that destructive interference produces a central value for $f_{n} / f_{p}$ which becomes large as the blind spot is approached (right plot of Fig. 2). This is reflected in the corresponding uncertainties shown in Fig. 3 (left plot), and highlights the need for complementary constraints from e.g. flavour observables (right plot).

\section{Summary}

An accurate evaluation of nucleon scalar matrix elements and their uncertainties remains an important ingredient in the interpretation of dark matter direct detection limits. For neutralino dark matter, it is well known [27] that the input value for $f_{s}^{N}$ can have a dramatic effect on excluded regions of the MSSM parameter space. Here we have highlighted another area where control over hadronic uncertainties is particularly important, namely, isospin violation in the vicinity of blind spots $[8,9]$. If signals of weak-scale supersymmetry remain persistently absent at the LHC and direct detection experiments, reducing these uncertainties will become particularly relevant as the MSSM is cornered in these regions of parameter space. 


\section{Acknowledgments}

LCT thanks the organisers and Hadron Structure \& Meson-Baryon working group of Chiral Dynamics 2015 for the opportunity to present this work. Financial support from the Swiss National Science Foundation (SNF) is gratefully acknowledged. AC is supported by a Marie Curie IntraEuropean Fellowship of the European Community's 7th Framework Programme under contract number (PIEF-GA-2012-326948).

\section{References}

[1] L. Bergstrom, Nonbaryonic dark matter: Observational evidence and detection methods, Rept. Prog. Phys. 63, 793 (2000) [hep-ph/ 0002126$].$

[2] K. A. Olive et al. [Particle Data Group Collaboration], Review of Particle Physics, Chin. Phys. C 38, 090001 (2014).

[3] K. Griest and D. Seckel, Three exceptions in the calculation of relic abundances, Phys. Rev. D 43, 3191 (1991).

[4] D. S. Akerib et al. [LUX Collaboration], First results from the LUX dark matter experiment at the Sanford Underground Research Facility, Phys. Rev. Lett. 112, 091303 (2014) [arXiv: 1310.8214$]$.

[5] E. Aprile [XENON1T Collaboration], The XENON1T Dark Matter Search Experiment, Springer Proc. Phys. 148, 93 (2013) [arXiv: 1206.6288$].$

[6] D. C. Malling et al., After LUX: The LZ Program, arXiv: 1110.0103.

[7] A. Crivellin, M. Hoferichter, M. Procura and L. C. Tunstall, Light stops, blind spots, and isospin violation in the MSSM, JHEP 1507, 129 (2015) [arXiv: 1503 . 034 78].

[8] C. Cheung, L. J. Hall, D. Pinner and J. T. Ruderman, Prospects and Blind Spots for Neutralino Dark Matter, JHEP 1305, 100 (2013) [arXiv : 1211 . 4873].

[9] P. Huang and C. E. M. Wagner, Blind spots for neutralino dark matter in the MSSM with an intermediate $m_{A}$ Phys. Rev. D 90, 015018 (2014) [arXiv: 1404 .0392].

[10] J. R. Ellis, A. Ferstl and K. A. Olive, Reevaluation of the elastic scattering of supersymmetric dark matter, Phys. Lett. B 481, 304 (2000) [hep-ph / 0001005 ].

[11] A. Corsetti and P. Nath, Gaugino mass nonuniversality and dark matter in SUGRA, strings and D-brane models, Phys. Rev. D 64, 125010 (2001) [hep-ph/ 0003186 ].

[12] J. R. Ellis, K. A. Olive and C. Savage, Hadronic Uncertainties in the Elastic Scattering of Supersymmetric Dark Matter, Phys. Rev. D 77, 065026 (2008) [arXiv : 0801 . 3656].

[13] V. Cirigliano, M. L. Graesser and G. Ovanesyan, WIMP-nucleus scattering in chiral effective theory, JHEP 1210, 025 (2012) [arXiv: 1205.2695].

[14] G. Colangelo et al., Review of lattice results concerning low energy particle physics, Eur. Phys. J. C 71, 1695 (2011) [arXiv: 1011.4408 ].

[15] B. Borasoy and U. G. Meissner, Chiral Expansion of Baryon Masses and $\sigma$-Terms, Annals Phys. 254, 192 (1997) [hep-ph/9607432].

[16] H. Y. Cheng, Low-energy Interactions of Scalar and Pseudoscalar Higgs Bosons With Baryons, Phys. Lett. B 219, 347 (1989). 
[17] J. Gasser, H. Leutwyler and M. E. Sainio, Sigma term update, Phys. Lett. B 253, 252 (1991).

[18] M. M. Pavan, I. I. Strakovsky, R. L. Workman and R. A. Arndt, The pion nucleon sigma term is definitely large: Results from a G.W.U. analysis of pion nucleon scattering data PiN Newslett. 16, 110 (2002) [hep-ph/0111066].

[19] J. M. Alarcón, J. Martin Camalich and J. A. Oller, The chiral representation of the $\pi N$ scattering amplitude and the pion-nucleon sigma term, Phys. Rev. D 85, 051503 (2012) [arXiv: 1110 . 3797].

[20] A. S. Kronfeld, Twenty-first Century Lattice Gauge Theory: Results from the QCD Lagrangian, Ann. Rev. Nucl. Part. Sci. 62, 265 (2012) [arXiv: 1203.1204 ].

[21] R. D. Young, Strange quark content of the nucleon and dark matter searches, PoS LATTICE 2012, 014 (2012) [arXiv:1301.1765].

[22] M. Hoferichter, J. Ruiz de Elvira, B. Kubis and U. G. Meißner, High-Precision Determination of the Pion-Nucleon $\sigma$ Term from Roy-Steiner Equations, Phys. Rev. Lett. 115, 092301 (2015) [arXiv:1506.04142].

[23] J. M. Alarcón, L. S. Geng, J. Martin Camalich and J. A. Oller, The strangeness content of the nucleon from effective field theory and phenomenology, Phys. Lett. B 730, 342 (2014) [arXiv: 1209.2870].

[24] O. Buchmueller et al., The CMSSM and NUHM1 after LHC Run 1, Eur. Phys. J. C 74, 2922 (2014) [arXiv:1312.5250].

[25] O. Buchmueller et al., The NUHM2 after LHC Run 1, Eur. Phys. J. C 74, 3212 (2014) [arXiv:1408.4060].

[26] P. Junnarkar and A. Walker-Loud, Scalar strange content of the nucleon from lattice QCD, Phys. Rev. D 87, 114510 (2013) [arXiv:1301.1114].

[27] J. Giedt, A. W. Thomas and R. D. Young, Dark matter, the CMSSM and lattice QCD, Phys. Rev. Lett. 103, 201802 (2009) [arXiv: 0907.4177 ].

[28] A. Crivellin, M. Hoferichter and M. Procura, Accurate evaluation of hadronic uncertainties in spin-independent WIMP-nucleon scattering: Disentangling two- and three-flavor effects, Phys. Rev. D 89, 054021 (2014) [arXiv: 1312.4951].

[29] G. Prezeau, A. Kurylov, M. Kamionkowski and P. Vogel, New contribution to wimp-nucleus scattering, Phys. Rev. Lett. 91, 231301 (2003) [astro-ph/0309115].

[30] V. Cirigliano, M. L. Graesser and G. Ovanesyan, WIMP-nucleus scattering in chiral effective theory, JHEP 1210, 025 (2012) [arXiv: 1205.2695].

[31] V. Cirigliano, M. L. Graesser, G. Ovanesyan and I. M. Shoemaker, Shining LUX on Isospin-Violating Dark Matter Beyond Leading Order, Phys. Lett. B 739, 293 (2014) [arXiv: 1311 . 5886].

[32] M. Hoferichter, P. Klos and A. Schwenk, Chiral power counting of one- and two-body currents in direct detection of dark matter, Phys. Lett. B 746, 410 (2015) [arXiv:1503.04811].

[33] CMS Collaboration [CMS Collaboration], Search for MSSM Neutral Higgs Bosons Decaying to Tau Pairs in pp Collisions, CMS-PAS-HIG-13-021.

[34] M. Misiak et al., Updated NNLO QCD predictions for the weak radiative B-meson decays, Phys. Rev. Lett. 114, 221801 (2015) [arXiv:1503.01789]. 